\title{
Modifications of Tanaka's Illuminated Contour Method
}

\section{Patrick Kennelly and A. Jon Kimerling}

\begin{abstract}
Visualization of topography can be greatly facilitated by the illuminated contour method. This method, popularized in a hand-drafted map by Tanaka, uses a gray background with black and white contours. A direction of illumination is assumed, and white contours represent illuminated topography, while black contours represent non-illuminated or shaded areas. Additionally, thickness of contours varies with the cosine of the angle between the azimuth of maximum slope (i.e., aspect) and the azimuth of illumination. We modified Tanaka's method by basing thickness of contour lines on twice the cosine of the angle between the surface normal and the illumination vector. The cosine of this angle is most commonly used in analytical hill shading. In addition, we present maps with changes in other visual variables and offer our evaluations. Lines with gray tones instead of black and white lines do not improve the illumination effect. We believe variations in the colors of contours and background with elevation can visually enforce information regarding topography. Our use of colors for aspect and variations in the width of contours for slope adds information to the map but does not assist with visualization of topography.
\end{abstract}

KEYWORDS: Illuminated contours, Tanaka method, relief contour method, analytical hill shading, aspect-slope map, MKS-ASPECT ${ }^{\mathrm{TM}}$, geographic visualization, Cascade Mountains, Mt. St. Helens, Mt. Adams, Washington state

\section{Introduction}

$\mathrm{C}$ ontour lines are an important cartographic tool used to symbolize continuous surfaces. In representing topography, contour lines allow quantitative measurements of elevation, slope, and relief. One criticism of the contour method, however, is that it does not present an easily recognized visual image of topography, especially to the untrained eye.

In 1950, Kitiro Tanaka promoted an innovative method to overcome this shortcoming. His technique assumed an illumination from the northwest (presuming a north-oriented map). He drew white contours to represent illuminated topography, and black contours to represent non-illuminated or shaded topography. All contours were drawn on a gray background to maximize visibility. He varied thickness of black and white contours based on the cosine of the angle $\theta_{\mathrm{T}}$ between two vectors (Figure 1). The first vector $\mathrm{A}$ was aspect of a surface, the azimuthal direction of maximum change in slope, and was given by a compass bearing on a horizontal plane. The second vector $\mathrm{I}_{\mathrm{A}}$ was the azimuthal direction of the illumination vector, also on a horizontal plane.

Patrick J. Kennelly, GIS Manager, Montana Tech of the University of Montana, Montana Bureau of Mines and Geology 1300 W. Park St. Butte, MT 59701. Phone: (406) 496-2986; Fax: (406) 496-4451. E-mail: <pkennelly@mtech.edu>. A. Jon Kimerling, Professor, Oregon State University, Department of Geosciences, Corvallis, OR 97331. Phone: (541) 737-1225; Fax: (541) 737-1200. E-mail: <kimerlia@geo.orst.edu>.
Tanaka applied a form of analytical hill shading to the contours, as if each contour line were a step on a model surface capable of being lighted from a given illumination direction. The thickness and spacing of black and white contours on a gray background created tonal variations that resulted in a three-dimensional shading effect.

Tanaka's method resulted in a strikingly threedimensional visualization of the topography of a rugged volcanic region of Kyushu, Japan (Figure 2). In his 1950 article, Tanaka called his technique "the relief contour method," although it is normally referred to as the "illuminated contour" or the "Tanaka" contour method (Imhof 1982). It should be noted that Tanaka was not the first to utilize this method, as several older maps, the first as early as 1870 , incorporated illuminated contours on a gray background (Imhof 1982). Tanaka, however, produced what has become a textbook example, accompanied by a detailed explanation of the theory and methodology.

In this paper, we propose variations of Tanaka's illuminated contour method. The first involves using a different angle to calculate contour thickness. This variation addresses a frequent criticism of Tanaka's method, that the topographic surface appears stepped or terraced, especially in areas of gentle gradient. We also suggest modifications in the gray tone of contours, the color of contours, and the color of the background. We present these to the reader with our impressions of their utility in enhancing data inherent in the contours, improving visualization of the topography, and present- 
ing additional data. We selected these variations from suggestions for improvement of illuminated contour maps by Imhof (1982), as well as from the appearance of frequently published examples of shaded relief, topographic, and slope-aspect maps.

Tanaka's map was meticulously and beautifully constructed by hand, using a method that was as quantitative as possible. This timerestrictive method has been automated by other authors (Gilman1973; Peucker et. al. 1975; Yoeli 1983; Eyton 1984), but always with the purpose of duplicating Tanaka's result as closely as possible. Here we suggest how our modifications can be implemented using Environmental Systems Research Institute's (ESRI) ARC/INFO Version 7.2.1 for UNIX software with the GRID module, or ArcView Version 3.2 for Windows with the Spatial Analyst extension. Both software combinations allow manipulations of raster and vector data necessary for our methods. We

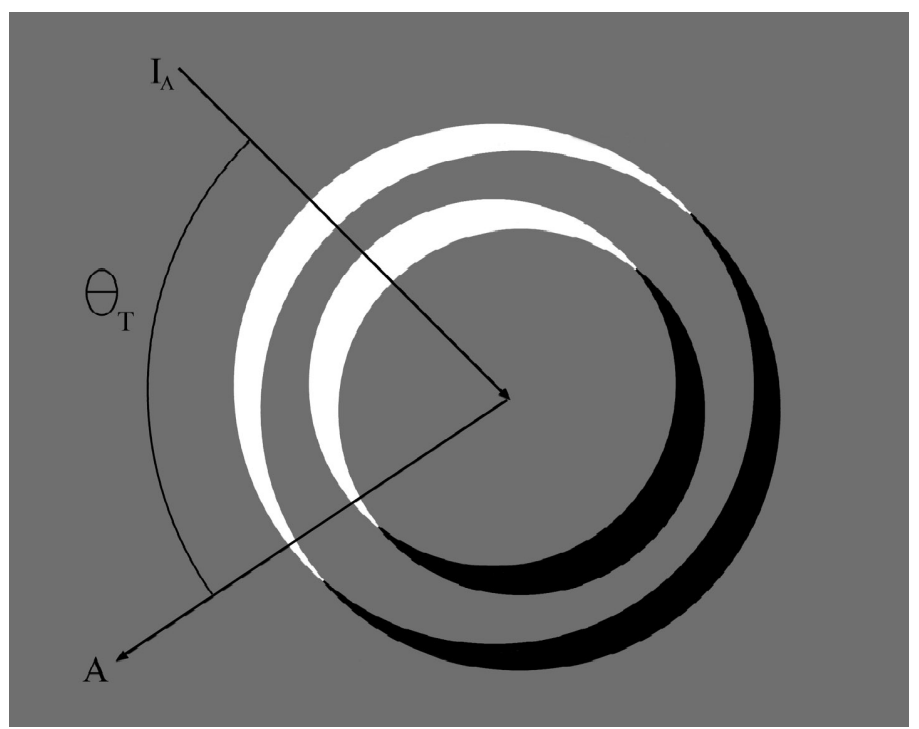

Figure 1. Azimuthal illumination $(\mathrm{I} A)$ and aspect $(\mathrm{A})$ vectors used to determine the thickness of contours with the Tanaka method. The thickness is proportional to the cosine of the angle $\theta_{\mathrm{T}}$ [ [Modified from Tanaka 1950; Figure 2 on page 445].

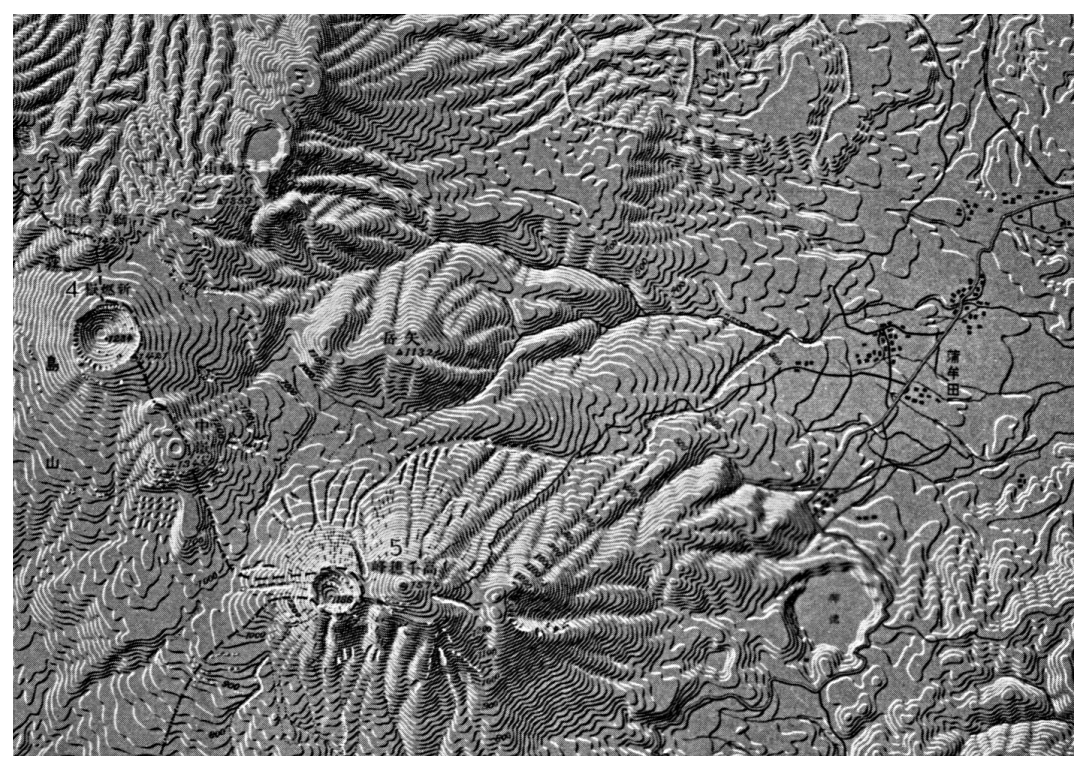

Figure 2. A portion of Tanaka's illuminated contour map of the Kirishima volcanic group near Kagoshima in Kyushu, Japan. [Reprinted from Tanaka 1950; Figure 7 on page 451].

discuss the general operations applied to the data below and present a step-by-step guide to the procedure in Appendix 1.

\section{Developments in Illuminating Contours}

The eyes of the cartographic world turned toward the Kirishima volcanic group near Kagoshima in Kyushu, Japan, in 1950, when Kitiro Tanaka cre- ated a contour map of this area with a three-dimensional appearance (Figure 2). Tanaka's method for drawing contours by hand is a marriage of cartography and calligraphy. He filed the tip of a drawing pen to the same thickness as that of the maximum contour thickness. Then, he held the pen at an orientation such that the broad edge of the filed tip of the pen was always parallel to the assumed horizontal illumination direction. The maximum thickness of the contour occurred at an aspect parallel to the illumination direction when the line thickness was equal to the width of the pen tip. The minimum thickness occurred at an aspect perpendicular to the illumination vector on the plane of the map. Everywhere between, the thickness of the contour was equal to the maximum thickness multiplied by the cosine of the angle $\theta_{\mathrm{T}}$ between the aspect of the topographic surface and the direction of illumination (Figure 1).

Tanaka realized that the resulting brightness and appearance of the illuminated contour map was merely an approximation of the brightness of a Lambertian surface, which is a theoretical surface that reflects all incident light and appears equally bright from all viewing directions (Horn 1982). 


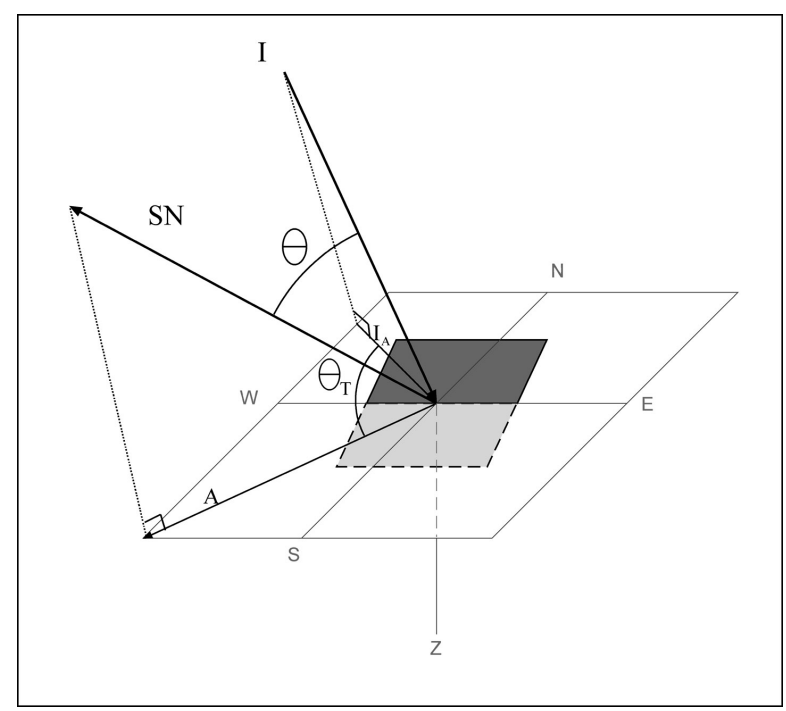

Figure 3. Illumination (I) and surface normal (SN) vectors in three dimensions used to determine the thickness of contours in this study. Line thickness is proportional to the cosine of twice the angle $\theta$. Vertical projections of vectors I and SN onto a horizontal plane result in Tanaka's vectors IA and A respectively. [Compare with Figure 1].

With such surfaces, brightness is proportional to the cosine of the angle $\theta$ between the illumination vector I and the surface normal vector SN (Figure $3)$. Unlike Tanaka's angle $\theta_{\mathrm{T}}$, this angle $\theta$ is not restricted to a horizontal plane but, rather, is defined between two vectors in three-dimensional space. If both the illumination vector and the surface normal vector were projected vertically onto the horizontal plane (i.e., the plane of the map), the angle between these two projected vectors would be Tanaka's angle $\theta_{\text {T. }}$ Many analytical hill shading and shaded relief maps are based on this Lambertian assumption.

Tanaka calculated the difference between the brightness of his illuminated contour method and hill-shading values for a Lambertian surface. Using an illumination vector with a zenith angle of $45^{\circ}$, he found no difference in brightness for surfaces sloping at $45^{\circ}$ in any aspect direction. For surfaces with gentle slopes, however, the differences in brightness approached 29 percent. Tanaka referred to this as the relative error in the brightness of his map.

The difference in brightness between Tanaka's illuminated contour map and the brightness of a map produced from the Lambertian assumption does not mean that Tanaka's technique is less effective. Horn (1982) pointed out that although most hill shading is based on the Lambertian assumption there is no evidence that this method optimizes perception of surface shape. To illustrate his point, Horn used a mathematical transformation he called a reflectance map to create hill-shading maps based on the Lambertian method, Tanaka's method, and several other methods. This allowed direct comparison of the three-dimensional shading effects resulting from brightness variations associated with Tanaka's method and a Lambertian model, as both now appeared as hill-shaded maps. Horn judged visualization of the map based on Tanaka's method to compare favorably to the Lambertian map.

Tanaka's "relative error in brightness" shows the robustness of hill-shading methods for visualization purposes. Tanaka pointed out that none of this variation associated with his "relative error" occurred with changes in aspect; all resulted from changes in slope for contour lines of the same aspect. Close examination of Tanaka's map in Figure 2 reveals this change in contour thickness with aspect only.

Tanaka's method has received some criticism regarding the appearance of the maps. Imhof (1982) noted that Tanaka's maps give the false impression of topography being stepped or terraced. This has been a common criticism of illuminated contours, especially in gently sloping terrain. Imhof also judged the transition from black to white contours to be too abrupt, leading to a loss of legibility. Using one circular contour as an example, Imhof suggested the following changes in the transition area between the black and white contour: varying gray tones, overlapping and tapering black and white lines, and using a dashed and/or dotted line.

Tanaka's illuminated contour method has been automated by several researchers. Usually, the automated methods have been computer based, but one of the earliest was photomechanical. Gilman (1973) attempted to use a prototype revolving illuminator to assist with the preparation of map copy for digitizing and the production of experimental slope maps for the U.S. Geological Survey. This photomechanical device could vary thickness of black and white contours with aspect direction for a given illumination direction to a tolerance of 0.2 inches. Gilman used the resulting illuminated contours as an intermediate step in his semi-automated relief shading process.

The advent of computer-assisted cartography allowed digital automation of the illuminated contour method. Peucker et al. (1975) replicated Tanaka's method with digital elevation data. For each contour line segment, the end points of the segment were used to calculate its direction, which was used to determine the thickness assigned to 
the line. To determine if the line should be colored black or white, the aspect direction was determined by looking at changes in slope in the $\mathrm{x}$ and y direction. Peucker revisited this method briefly in 1980, noting that it adds very little computing time to conventional digital contouring.

Shaded or shadowed contours (Figure 4) are similar to illuminated contours, but they are all black and vary in thickness for contour line segments on non-illuminated surfaces only (Bertin 1981; Imhof 1982; Robinson et al. 1995). As all contours are black, a gray background is not necessary. Imhof indicated a number of deficiencies in this method, including a lack of visual balance, a layered appearance in gently sloping to flat areas, and coalescing contours on steep slopes.

Yoeli (1983) derived a computer algorithm for generating such shadowed contours through the use of a pen plotter. Yoeli's program interpolated contours as a spline curve from a digital terrain model. Yoeli used a pen plotter to plot the contours, systematically displacing the origin of the drawing in the assumed direction of illumination and redrawing all contours that would be on the shaded side several times. He used the DEM (Digital Elevation Model) grid cell values from which the contour lines were interpolated to determine which contours were to be shaded. Although this method is similar to that used by Peucker et al. (1975), Yoeli (1983) was able to simplify the process by using gridded DEM data to determine shaded and non-shaded contours.

Eyton (1984) developed raster-contouring algorithms that allowed the creation of illuminated contour maps drawn with DEM grid cells. He used slope and aspect data derived from the DEM to illuminate individual grid cells. Contour lines were composed of cells on the edge of elevation class boundaries. Line thickness, however, was not varied with aspect with this method; all contour lines were one grid cell thick. The method, nevertheless, resulted in a map with a three-dimensional appearance, further evidence of the robustness of Tanaka's method.

\section{Our Modifications to Tanaka's Method}

Tanaka understood that his illuminated contour map was an approximation of brightness associated with hill shading under the Lambertian assumption; however, no simple method of drawing contours would account for changes in both slope and aspect. In relating all changes in contour thickness to the aspect vector, Tanaka and other

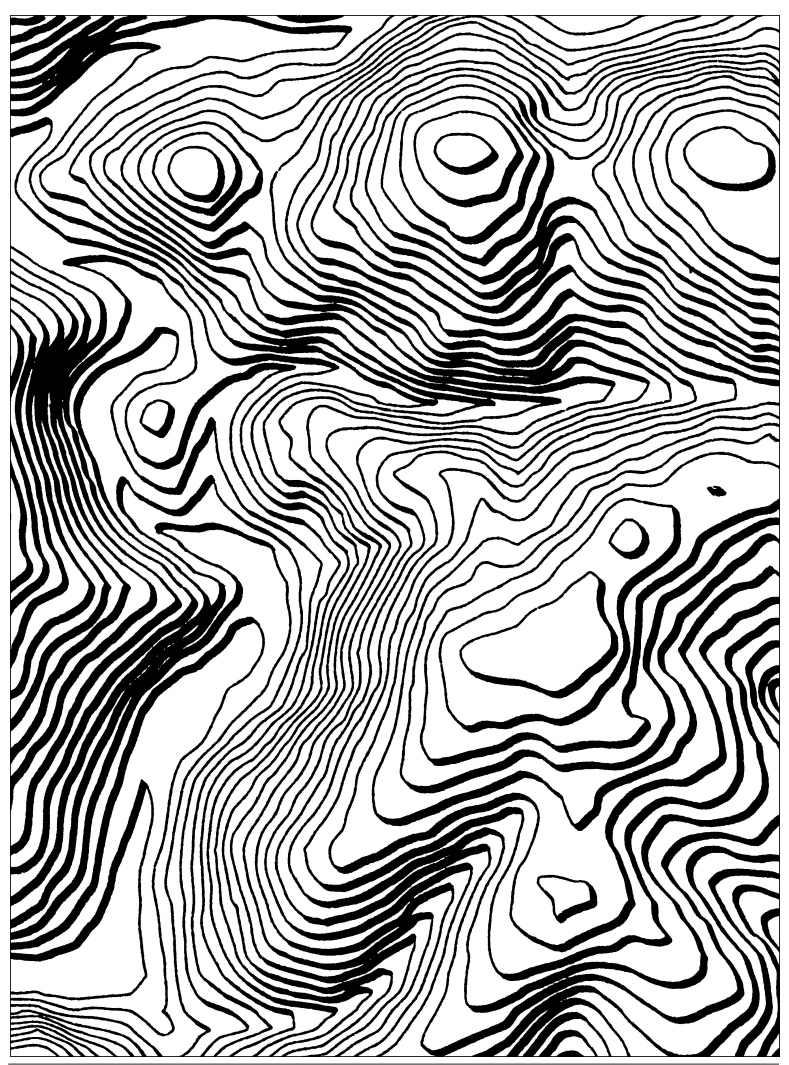

Figure 4. A portion of a computer generated shadowed or shaded contour map. [Reprinted from Yoeli 1983; Figure 18 on page 109].

cartographers have been able to employ simple construction methods. Tanaka (1950) was able to hold his drafting pen at a constant angle, and Yoeli (1983) was able to shift the origin of his computer-plotted map to thicken contours appropriately. The data not captured with their methods but captured in our modifications are variations in illumination resulting from changes of slope for contour line segments with a constant aspect.

To allow a comparison of these methods, we have created illuminated contour maps following the method outlined by Tanaka and using our modified method. Our modified illuminated contour maps account for variations in line thickness based on a hills-hading value related to $\theta$ (Figure 3 ), a fundamental change in the method. We also vary the grayness and color of contour lines, as well as the color of the background and contour lines to allow readers direct comparison of these visual variables.

To illustrate our methods, we utilize elevation data from a U.S. Geological Survey 1:250,000 DEM for the Cascade Mountains of Oregon and Washington. The grid cells measure approximately 90 square meters. Two-hundred-meter contours were derived from the DEM. The extent of the analysis 


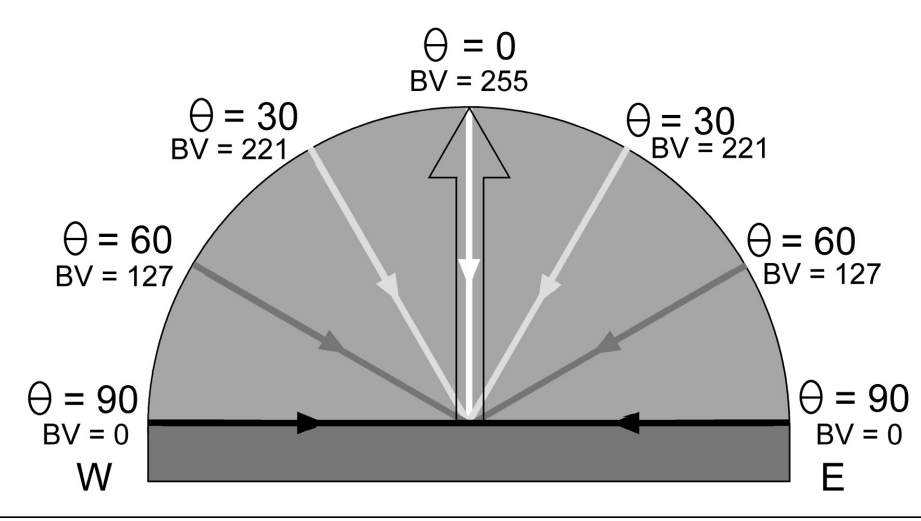

Figure 5. The relationship between the surface normal vector, the illumination vector, $\theta$, and the hillshading brightness value (BV $=255{ }^{*} \cos$ $(\theta)$ ). Values of $\theta$ fall between $0^{\circ}$ and $90^{\circ}$, and more than one illumination vector can have the same value of $\theta$ and $B V$.

is an area of about $180 \mathrm{~km} \times 380 \mathrm{~km}$ centered on the Cascade range, with a northern boundary on the north slope of Mt. Rainier and a southern boundary just south of Mt. Hood. We selected this area for its frequent changes in topographic aspect and slope. Also, this area, which includes the Mt. St. Helens caldera, is similar in topography to the volcanic landscape first mapped with illuminated contours by Tanaka. All statistics reported in this paper are for this area. All figures in this paper, however, display a smaller area, which includes Mt. St. Helens and Mt. Adams, with an approximate extent of $75 \mathrm{~km}$. x $35 \mathrm{~km}$.

We calculated relative brightness for each grid cell of this DEM, using the Lambertian assumption (Figure 3). We chose the direction of illumination to have an azimuth of $315^{\circ}$ (from the northwest) and an inclination of $45^{\circ}$ from a horizontal plane. The resulting initial hill-shading values will vary between $1\left(\cos 90^{\circ}\right)$ and $0\left(\cos 0^{\circ}\right)$. The hill-shading value is then normalized to a value between 0 and 255, the range of brightness values for display.

Figure 5 illustrates the relationship between the angle $\theta$ and the hill-shading brightness value (BV). Figure 5 shows a cross section of a flat surface. The unfilled arrow is the surface normal. The surface is illuminated from a number of directions, indicated by the black, white, and gray arrows. The angle between the surface normal vector and the illumination vectors is $\theta$. The value of $\theta$ must be less than $90^{\circ}$; at angles greater than $90^{\circ}$ the illumination vector will no longer strike the surface. The brightness value for hill shading is $(\mathrm{BV}=\cos (\theta)$ *255), which corresponds to the grayness of each illumination vector. The values of $\theta$ and $\mathrm{BV}$ are not unique. In this example, all values of $\theta$ (except $\theta=0$ ) are defined by two illumination vectors.
In three dimensions, a constant value of $\theta$ would define a cone of illumination vectors around the surface normal vector. A similar relationship exists if the illumination vector is held constant and the surface normal vector is allowed to vary.

We separated the hill-shadings values into classes, with each class corresponding to a change in $\theta$ of $5^{\circ}$ (Figure 6). Because most grid cells had a slope of less than $5^{\circ}$ and the illumination vector had an inclination of $45^{\circ}$, the majority of cells fell into two classes with values of $\theta$ between $40^{\circ}-45^{\circ}$ and $45^{\circ}-50^{\circ}$. Although 18 (i.e., $90^{\circ} / 5^{\circ}$ ) potential classes exist, data fell into 12 classes centered around an angle of $45^{\circ}$; there were no grid cells with surface normal vectors at an angle greater than $75^{\circ}$ or less than $15^{\circ}$ to the illumination vector in the study area.

We reclassified the data into these 12 groups. Next, we converted the reclassified grid into a polygon coverage. Each polygon outlined reclassified grid cells of the same hill-shading value range. Then we intersected the contours with the polygons encoded with the hill-shading values, thus transferring the hill-shading values to each intersected contour line segment. We then illuminated the contours by applying black and white colors, as well as changing the thickness of all contour line segments. The thickness of the black and white lines varied as a function of $\cos (2 \theta)$. We decided to use this value instead of $\cos (\theta)$ to achieve visual balance in the map (see Appendix). We used a gray background with a moderate brightness value of 125 out of 255 . The resulting map is presented at a scale of 1:500,000 as Figure 7.

We also created an illuminated contour map of the same area using Tanaka's method for comparison with our method. This procedure was similar to the one outlined above. The only difference was that classes were based on the angle $\theta_{\mathrm{T}}$ derived from the DEM instead of an angle $\theta$. Unlike $\theta, \theta_{\mathrm{T}}$ varies from $0^{\circ}$ to $360^{\circ}$, based on the angle between the illumination vector and an azimuth vector. Values of $\theta_{\mathrm{T}}$ were reclassified into $45^{\circ}$ intervals, converted to a polygonal coverage, intersected with the contour coverage, and used to assign appropriate contours to black and white, as well as to adjust contour thickness.

The use of a $45^{\circ}$ classification for $\theta_{\mathrm{T}}$ deserves further discussion. $\theta_{\mathrm{T}}$ can have values ranging from $0^{\circ}$ to $360^{\circ}$. Defining classes at $45^{\circ}$ intervals creates eight separate categories, as well as a ninth category for flat-lying areas. An important observation of the eight non-flat classes is that the lengths of 
contour lines are similar in all eight classes. $\theta$ can have values ranging from $0^{\circ}$ to $90^{\circ}$. In many cases, however, all of these values are not present in a given area. Furthermore, in this study area, the distribution of $\theta$ is strongly centered at $45^{\circ}$. Although the values of contour line segments vary from $15^{\circ}$ to $75^{\circ}$, 99.7 percent of the line segments by length are between $30^{\circ}$ and $60^{\circ}$ (Figure 6). This means that there are effectively only six significant classes (and corresponding changes in black and white contour width) in Figure 7. This should compare favorably with the eight significant classes for the Tanaka illuminated contour map in Figure 8.

The most salient difference between Figures 7 and 8 is that line thickness varies with changes in slope in Figure 7 but not in Figure 8. Mt. Adams, on the right side of the figures, offers a clear example. In Figure 7, the contours on the distal northwestern flank of Mt.

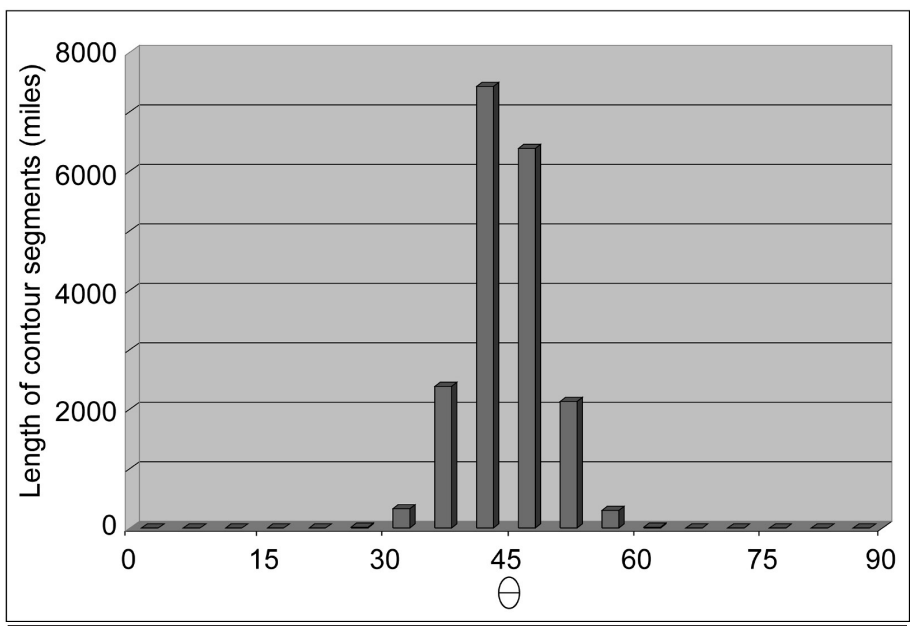

Figure 6. Distribution of the angle $\theta$ versus length of contour lines for the entire study area. The distribution is centered at angles of $\theta$ between $40^{\circ}$ to $50^{\circ}$, resulting from our use of an illumination vector with an inclination of $45^{\circ}$ from a horizontal plane, and most topography having a slope of less than $5^{\circ}$.

Adams are thinner than the same contours in Figure 8. We suggest that thinner contours in gently sloping terrain help to eliminate some of the stepped or terraced appearance of topography associated with Tanaka's method. The steep eastern slope near the crest of Mt. Adams is also apparent in Figure 7. In Figure 8, contour thickness in this area is a function of aspect only, with thickest black contours always indicating a southeast aspect.

We used our modified illuminated contour method for all of the following maps presented in this paper. Our first variation used contours with a constant thickness $(1.5 \mathrm{pt} .=3 / 64$ inch $=1.2$ millimeters), while changing the grayness assigned to individual contours (Figure 9). This is similar to the gray transition described by Imhof (1982), which he believed would help to overcome the abrupt transition from black to white. This technique is similar to changing gray tones on a hill-shading or shaded relief map.

We used a background brightness value for Figure 9 of 90/255 instead of the 125/255 value used for Figures 7 and 8 to avoid the majority of contours having little contrast with the background. For example, if a brightness value of 120 were used for the background, all contour segments with a hillshading angle between $40^{\circ}$ and $45^{\circ}$ (representing 39.1 percent of the total contour length would have no contrast with the background. In contrast, using a value of 90 (only 12.6 percent of the total contour line length) is close to background gray.

Unfortunately, this technique does not improve the appearance of the illuminated contour map. The overly abrupt change from black to white con-

tours that Imhof (1982) noted in illuminated contour maps has been eliminated, but the resulting map appears overly softened or even out of focus. This result, however, varies grayness along extensive lengths of contour lines, a more gradual or less sharp black to white transition than the narrow zone proposed by Imhof in his schematic contour example.

All of Imhof's ideas for a narrow transition zone (including changing gray values, tapering and dashing contours) could not be effectively implemented with our illumination method. Imhof's concept would require the use of at least two of our classes, one transitioning from black into gray and the other from white into gray. The two required classes would be associated with $\theta$ values between $40^{\circ}$ and $50^{\circ}$. As this interval represents 72.7 percent of the total contour length (Figure 6), the resulting maps did not reflect Imhof's conceptual drawings with a narrow gray transition zone. This is apparent in Figure 9, as the majority of contour segments are a moderate gray, with a small minority of contour segments approaching black or white. Imhof's concept would be best implemented with Tanaka's method and additional classes represented by small changes in $\theta_{\mathrm{T}}$ in the transition zone from black to white contours.

This study added color to illuminated contour maps using two methods. First, color was used for coloring contours and background with changes in elevation in an attempt to reinforce the quantitative data present in the contours. Second, we used luminance of colors and line thickness to simultaneously display slope and aspect. 


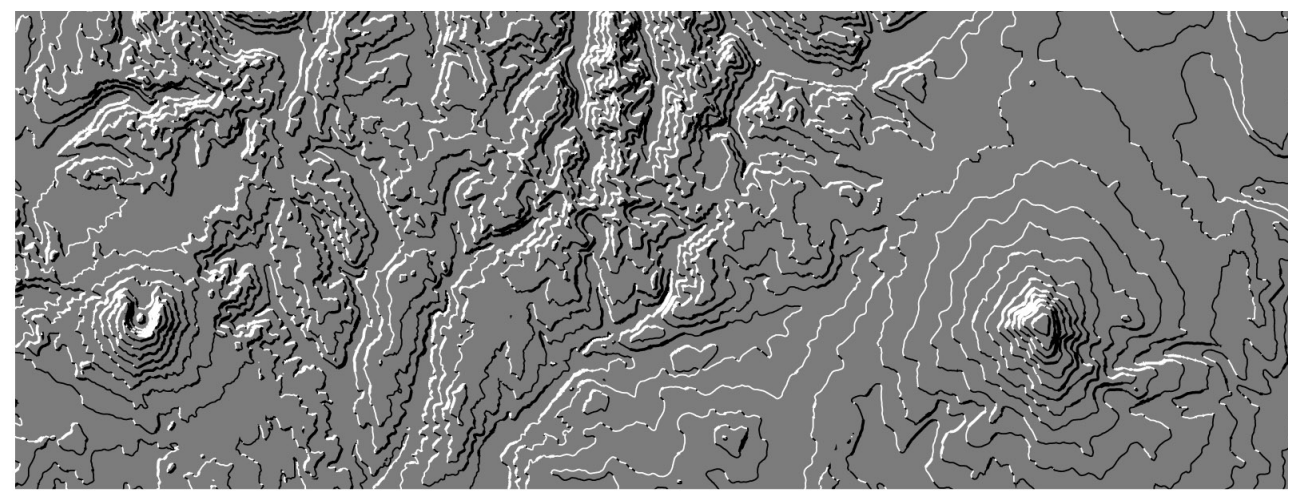

Figure 7. Illuminated contour map created by the modified Tanaka method described in this study, which assigns contour thickness based on the angle $\theta$.

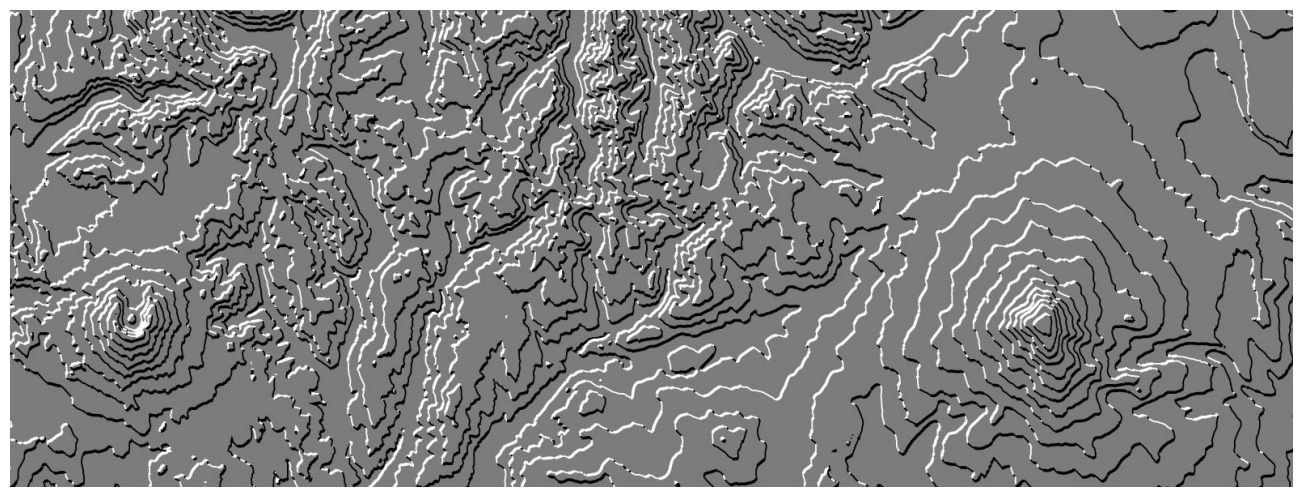

Figure 8. Illuminated contour map created by the Tanaka method, with contour thickness based on the angle $\theta_{\mathrm{T}}$

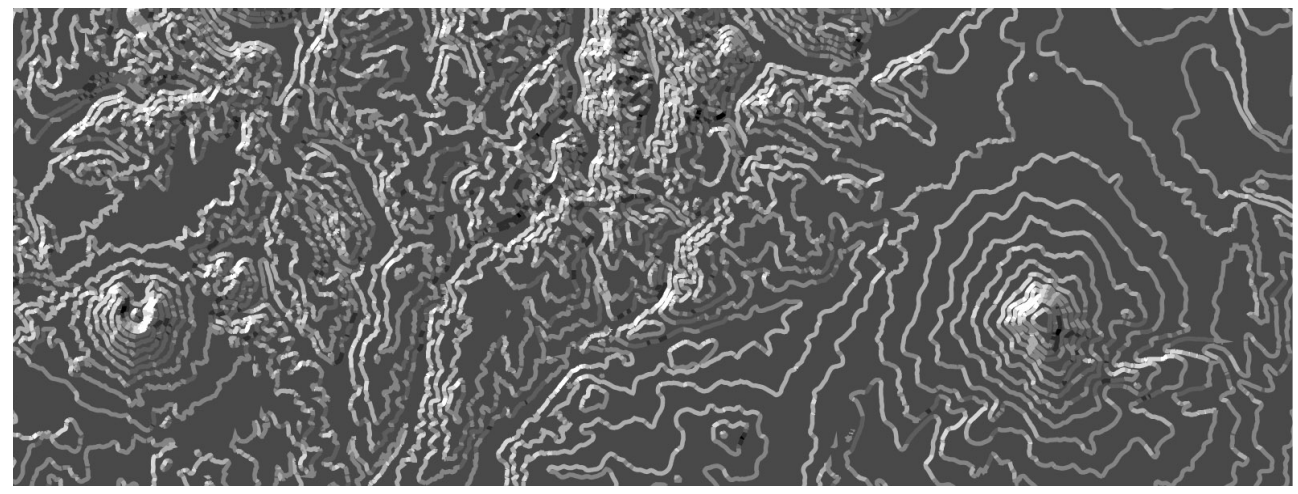

Figure 9. Illuminated contours using constant line thickness, butvarying shades of gray as a function of $\theta$.

We applied color to elevation in two ways. With our first method (Figure 10), we drew black and white illuminated contours on a layertinted background based on the Hue-SaturationValue(Brightness) or HSV color model. In Figure 10 , the hue and saturation change between every contour elevation value. Hues and saturations were selected to give the topography a somewhat realistic appearance, and are similar to colors popular in shaded relief maps. All colors have the same brightness value equal to $150 / 255$, which is greater than the gray used in Figures 7 and 8, as the colors help provide contrast to the black and white contours.

We believe the resulting map aids the user in determining elevation, because color reinforces the trends in elevation (i.e., up versus down) seen in the illuminated contours and facilitates the quick comparisons of absolute elevations in non-adjacent areas. This technique does not offer all the detail of a shaded relief map, as analytical hill shading is applied in a linear versus area-wide fashion. It does, however, allow easy recognition of landforms and elevation as well as the quantitative measures of elevation data (although our suppositions await user testing).

With our second method, we apply colors directly to the illuminated contours and keep the background a constant gray. Contours are now assigned the same hue and saturation values used with layer tinting in Figure 10. We vary the thickness of contours as in previous examples, but we also vary the brightness of the contour color with respect to illuminated or non-illuminated topography (Figure 11). Brightness values are 255/255 for illuminated color contours and 100/255 for nonilluminated color contours. 
We believe this approach also enhances elevation information in a manner similar to that discussed for Figure 9. In addition, the smaller variation in brightness between illuminated and nonilluminated contours (100-255 for color contours vs. $0-255$ for black and white contours) provides a less abrupt transition than in Figure 7. Our map gives a less stark and, consequently, subtler effect than Tanaka's method or our modified method in black and white. In areas of gentle slope or complex topography, however, this subtle effect may be difficult to discern.

The analytical hill-shading technique we used in all of our maps provided data on the orientation of a surface in three dimensions; however, hill-shading brightness values or the angle $\theta$ do not uniquely define the orientation of that surface (see Figure 5). Given a constant illumination vector, a multitude of surface normal vectors defining a cone will all correspond to a given value of $\theta$ in three-dimensional space. We can, however, specify the orientation of a surface in three dimensions by using two unit vectors (Horn 1982). An example of such vectors would be a unit vector in the direction of aspect and a unit vector with the inclination from a horizontal plane of slope.

We reclassified the aspect grid into eight classes representing $45^{\circ}$ intervals of aspect, with flat topography represented by a ninth. We also reclassified the slope grid into six classes representing $5^{\circ}$ intervals of slope. These two reclassified grids were then converted to polygons, which were intersected with each other, and finally intersected with the original contour coverage. The resulting coverage was comprised of more than three times (250,000 vs. $75,000)$ the number of contour line segments as the coverage created from the hill-shading intersection used for Figure 7. Although greater storage is required for this method, we can now show changes in slope and aspect separately.

Figure 12 is the resulting illuminated contour map based on both slope and aspect. The colors are based on the Moellering and Kimerling (1990) MKS-ASPECT $^{\mathrm{TM}}$ method. This opponent process color scheme uses the Hue-Lightness-Saturation (HLS) color system and serves two purposes. First, it simulates relief shading by using colors with relative luminance values that fit a shading curve based on a modified cosine function. Second, it maximizes visual discrimination between the eight classes to optimize legibility. In addition to using the MKS-ASPECT ${ }^{\mathrm{TM}}$ color scheme for aspect, we vary thickness of the contours with slope; thicker contour lines are associated with steeper slopes.
Varying map symbols with slope and aspect simultaneously is not a new concept. Hachure maps vary line orientation and thickness with aspect and slope respectively (Imhof 1982). In a more recent innovation, Brewer and Marlow (1993) created a hill-shading method in which color varied with changes in slope and aspect. In their model (based on the Hue-ValueChroma (HVC) color system), hue varied with aspect, and value and chroma varied with slope. In contrast, our method varies luminance with aspect and contour thickness with slope.

We recognize that there are two separate issues concerning Figure 12. One issue is the use of the MKS-ASPECT ${ }^{\mathrm{TM}}$ method to increase information content; the other issue is its use as a visualization tool. More information on surface orientation is displayed in Figure 12 than in any other map in this paper. Tanaka's method (Figure 8) displayed information on aspect only. Figure 7 displays information related to the orientation of the surface in three dimensions, but slope and aspect were indivisibly married in the single hill-shading value defining black and white color and thickness. Figure 12 displays slope and aspect separately, providing an increase in information content in the map.

Admittedly, Figure 12 is complex, but we feel it helps to clarify data that could be ambiguous in all the other maps we have shown. With Tanaka's method and our modified Tanaka method, contours with northeast and southwest aspects would have the same line thickness and color (Figures 7 and 8). This is not an issue on easily recognized landforms such as Mt. Adams, where up and down directions are easily discernible. In more complex topography, however, aspect cannot easily be discerned by Tanaka's method or our modified method, but it can be easily differentiated by the changes in color of the contours in Figure 12.

Although more information is displayed in Figure 12 than in Figure 7, we find the increase in information does not facilitate our visualization of landforms. Maps derived from hill-shading techniques seem to have a visual effect that is difficult to surpass, even using additional information on the orientation of the surface not available from hill-shading data.

\section{Conclusions}

When Tanaka produced his illuminated contour map in 1950, it was not possible to derive a simple construction method that would account for all theoretical brightness variations associated with 


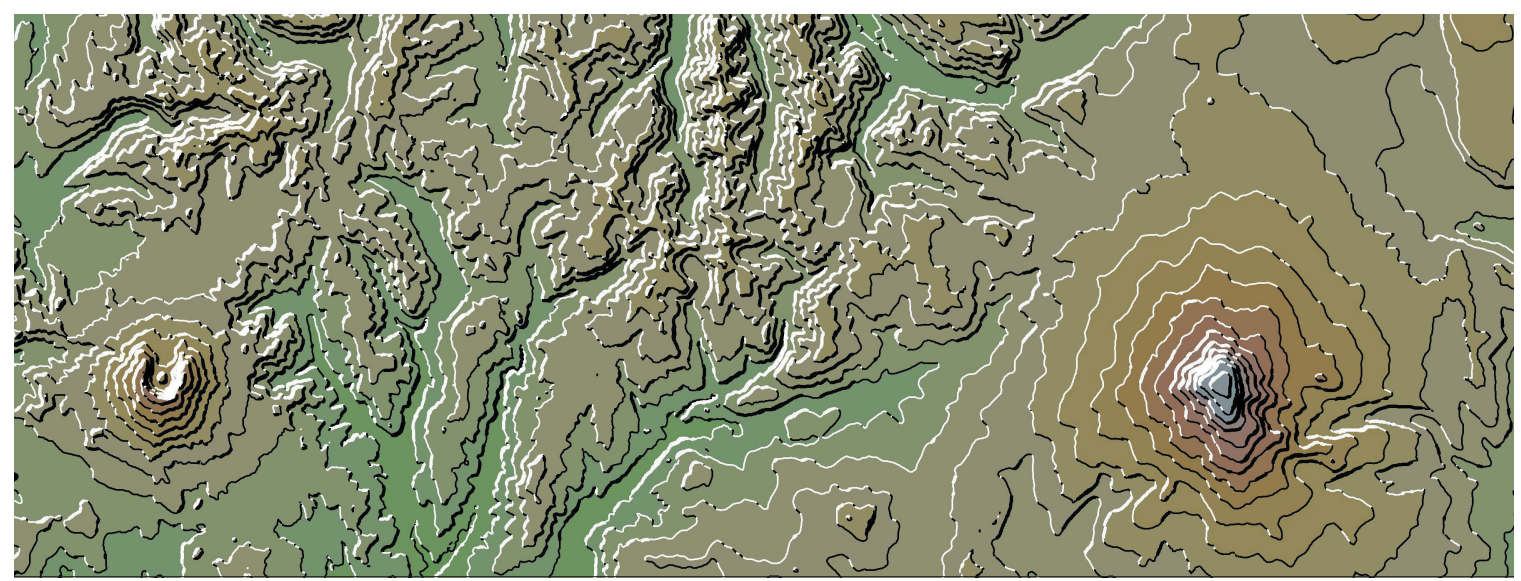

Figure 10. Illuminated contours with layer tints applied to the background based on elevation. Each background interval between contours has a unique color based on variations in hue and saturation designed to give the topography a somewhat realistic appearance.

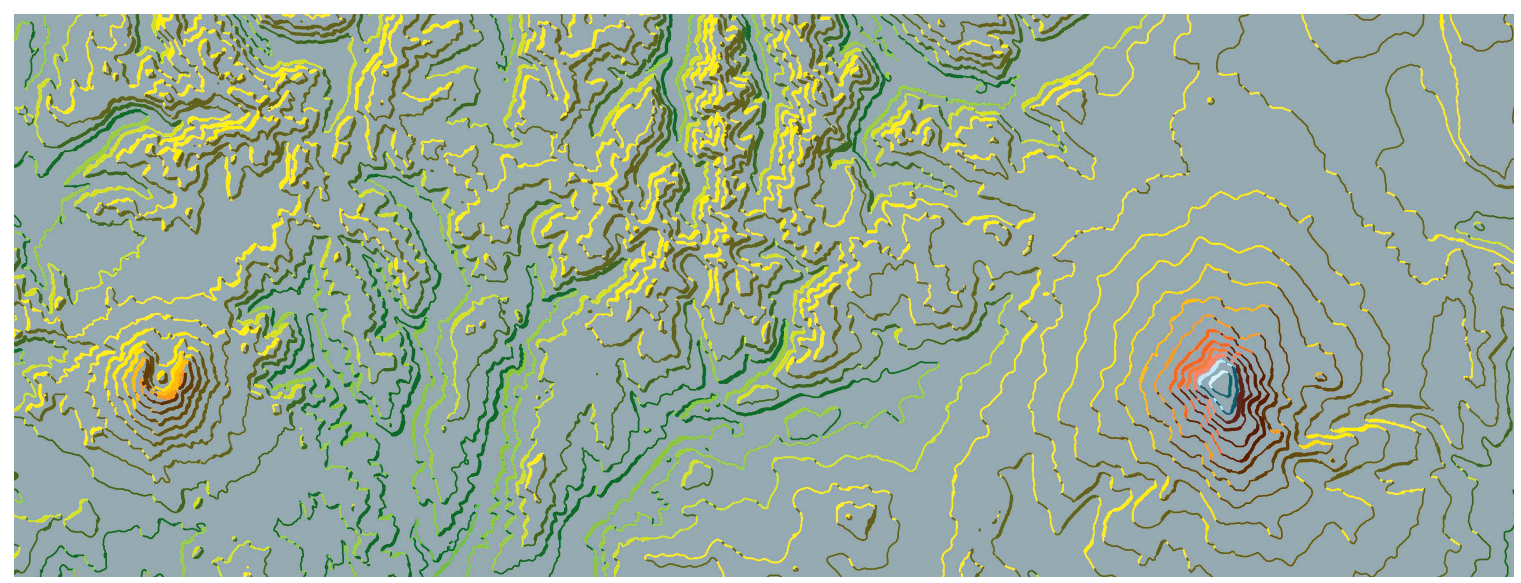

Figure 11. Illuminated contours with colors applied to contours based on elevation. Each contour level has a unique color based on the same variations in hue and saturation used in Figure 10. Brightness of the contour colors varies with illumination.

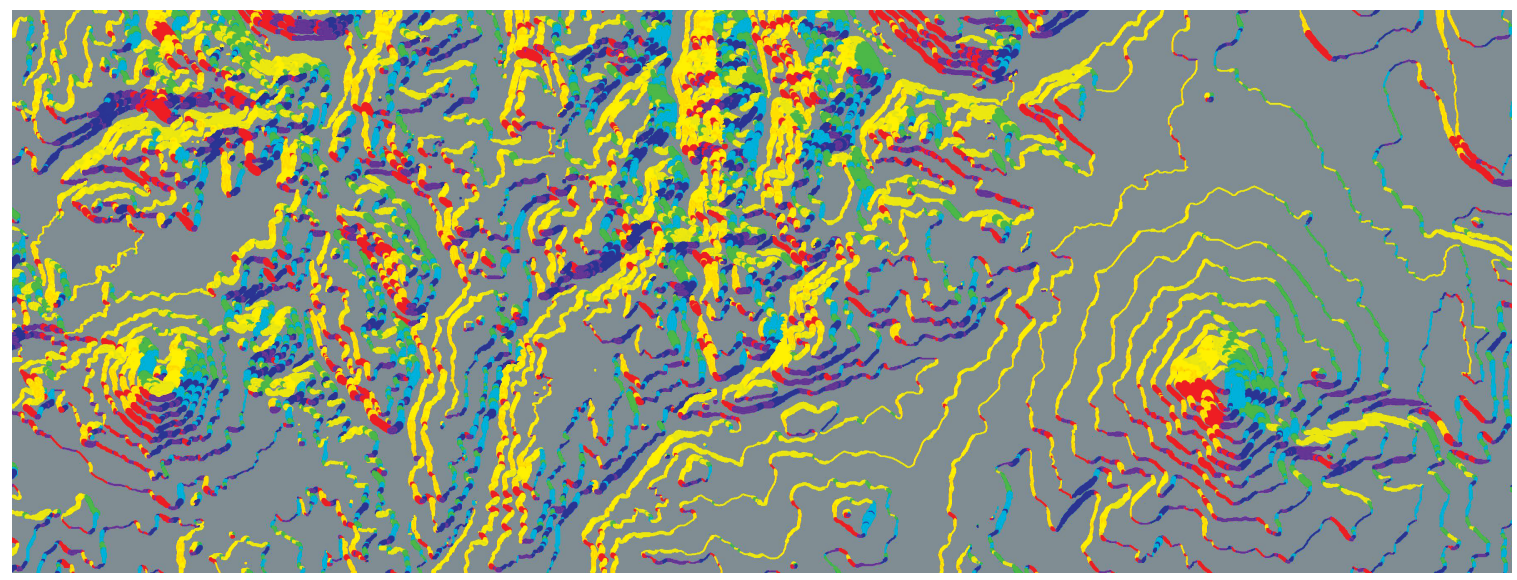

Figure 12. Illuminated contours using variations in the color and thickness of lines. The color of contour lines varies with aspect and is based on the MKS-ASPECTTM color scheme of Moellering and Kimerling (1990). MKSASPECTTM is protected by two patents, No. 5,067,098 and No. 5,283, 858, and formal property of The Ohio State University Research Foundation. The thickness of contour lines varies with slope. 
the Lambertian assumption. In addition, a systematic study of variations in visual properties associated with these contours was time-prohibitive. With computer automation, we have easily and systematically varied visual components of an illuminated contour map.

The most significant difference in methodology between this study and previous ones is that we have calculated an illumination factor based on three-dimensional surface normal and illumination vectors rather than using aspect and illumination vectors on a two-dimensional plane. This difference allows contours of the same aspect to vary with topographic slope. The resulting maps also have less of a stepped or terraced appearance thanTanaka's original map.

Our other changes to illuminated contour maps are more subjective in their merit. Using shades of gray instead of using changes in line thickness produces a less abrupt black-to-white transition, but the contours appear to be less sharply defined, even out of focus. This and other changes designed to make the transition from black to white contours less abrupt are not appropriate for illuminated contours using our modified method, but they could be used for illuminated contours in Tanaka's original method.

We applied colors based on elevation to both the background and to illuminated contours. This technique visually underscores the relief and elevation defined by the illuminated contours. Applying layer tinting to the background allows easy comparisons of elevation. Applying colors to contours enabled us to produce a less stark illuminated contour map, with a less abrupt transition between illuminated and non-illuminated contours.

Finally, by applying appropriate colors to contours based on aspect and varying contour thickness based on slope, we increased the information content of the map. Changes in slope and aspect uniquely define the orientation of each contour segment in three-dimensional space. We represented these changes by contour thickness for slope and color for aspect. We do not, however, see improvements in visualization of the topography based on this method.

In summary, we offer an alternative simple method for creating illuminated contours, which provides a strikingly three-dimensional look. We offer suggestions for methods to emphasize information contained in illuminated contour maps, as well as enhance the overall appearance of the map. Although no method is optimal, this study outlines experimental alternatives. The appropriate method selected by the cartographer will depend on the desired visual focus of the resulting product and will ultimately need to be tested with users.

\section{ACKNOWLEDGEMENTS}

We would like to thank Dr. Harold Moellering for permission to use the MKS-ASPECT ${ }^{\mathrm{TM}}$ color scheme for Figure 12. MKS-ASPECT ${ }^{\mathrm{TM}}$ is protected by two patents, No. 5,067,098 and No. 5,283, 858, and it is a formal property of The Ohio State University Research Foundation.

\section{REFERENCES}

Bertin, J. 1981. The semiology of graphics. Translated by William A. Berg. Madison, Wisconsin: University of Wisconsin Press.

Brewer, C. A., and K. A. Marlow. 1993. Computer representation of aspect and slope simultaneously. In: Proceedings, Eleventh International Symposium on Computer-Assisted Cartography (Auto-Carto-11), Minneapolis, Minnesota. pp. 328-37.

Eyton, J. R. 1984. Raster contouring. Geo-Processing 2: 221-42.

Gilman, C.R. 1973. Photomechanical experiments in automated cartography. Journal of Research of the U.S. Geological Survey 1: 223-7.

Horn, B. K. P. 1982. Hill shading and the reflectance map. Geo-Processing 2: 65-146.

Imhof, E. 1982. Cartographic relief presentation. Berlin and New York: Walter de Gruyter.

Moellering, H., and A. J. Kimerling. 1990. A new digital slope-aspect display process. Cartography and Geographic Information Systems 17: 151-9.

Peucker, T. K. 1980. The use of computer graphics for displaying data in three dimensions. Cartographica 17: 59-72.

Peucker, T. K., M. Tichenor, and W. D. Rase. 1975. The computer version of three relief representations. In: Davis, J. C., and M. McCullagh (eds), Display and analysis of spatial data. New York, New York: John Wiley and Sons. pp. 187-97.

Robinson, A. H., J. L. Morrison, P. C. Muehrcke, A. J. Kimerling, and S. C. Guptill. 1995. Elements of cartography, $6^{\text {th }}$ ed. New York, New York: John Wiley and Sons.

Tanaka, K. 1950. The relief contour method of representing topography on maps. Geographical Review 40: 444-56.

Yoeli, P. 1983. Shadowed contours with computer and plotter. The American Cartographer 10: 101-10. 


\section{Appendix}

The following are the ARC/INFO Version 7.2.1 commands and ArcView Version 3.2 menu choices we used to create Figure 7. Commands for producing other figures are available from the authors. It is necessary to have the GRID module for ARC/INFO and the Spatial Analyst extension for ArcView loaded to perform these operations.

\section{ARC/INFO Procedure}

1)Create 200m. contours from the DEM (named elev in this example)

Arc: Grid

Grid: cntr $=$ contour (elev, interval, 200)

2) Create hillshading polygons representing equal $5^{\circ}$ increments of the angle $\theta$.

Grid: hlshd $=$ hillshade (elev, 315,45 , shade)

Grid: hlshd $1=$ float (hlshd)

Grid: hlshd $2=$ hlshd $1 / 255$

Grid: theta $\operatorname{rad}=\operatorname{acos}$ (hlshd2)

Grid: theta $=$ theta_rad $*$ deg

(Deg is a built-in constant to convert radians to degrees, or use 57.29578 degrees/radian)

Grid: rcls $=$ reclass (theta, remap.txt)

(Remap.txt is a text file containing ranges of values of $\theta$, and the value to which these ranges will be reclassified.)

Remap.txt file format

$$
\begin{array}{cccc}
0 & 5 & : & 1 \\
5 & 10 & : & 2 \\
\ldots & \ldots & \ldots \\
80 & 85 & : 17 \\
85 & 90 & : 18
\end{array}
$$

Grid: hs_poly $=$ gridpoly $(\mathrm{rcls})$

Grid: $q$

$3)$ Intersect the contours with the hillshading polygons to transfer values of $\theta$ to the contour line segments.

Arc: intersect cntr hs_poly illum line

Resulting coverage is illum

4)Apply legend to the illuminated contour coverage (illum)

(See "About the Legend" below.)

\section{ArcView Procedure}

1)Load necessary extensions and DEM data

File: Extensions:

Spatial Analyst

Geoprocessing

View: Add Theme

Data source type: Grid data source Add DEM data
2) Create contours from the DEM

Surface: Create contours

Contour Parameters

Contour interval: 200

Base contour: 0

3) Create hillshading polygons representing equal

$5^{\circ}$ increments of the angle $\theta$.

Surface: Compute Hillshade

Compute hillshade:

Azimuth: 315

Altitude: 45

(N.B. This is the only step in ArcView that does not offer all of the flexibility of similar ARC/INFO commands. Shading as well as shadowed grid cell values are calculated, with shadowed grid cells given a "hillshading" value of 0 . Shadowing can be prevented by choosing an illumination vector with an altitude (inclination) greater than the steepest slope.)

Analysis: Map Calculator

Map Calculation 1:

( [Hillshade of Elev] ).Float / 255)

Analysis: Map Calculator

Map Calculation 2:

([Map Calculation 1].Acos) * 57.29578

Analysis: Reclassify

Reclassify Values

Old Values New Values

$\begin{array}{ccc}0 & 5 & 1 \\ 5 & 10 & 2 \\ \ldots & \ldots \ldots & \\ 80 & 85 & 17 \\ 85 & 90 & 18\end{array}$

Select Reclass of Map Calculation 2

Theme: Convert to Shapefile

Name: hs_poly

4) Intersect the contours with the hillshading polygons to transfer values of $\theta$ to the contour line segments.

View:GeoProcessing Wizard

Geoprocessing Intersect two themes

1) Select input theme to intersect: Contours of Elev

2) Select an overlay theme: hs_poly

Resulting shapefile: Intsct1.shp

5) Apply legend to the illuminated contour shapefile (Intsct 1.shp)

(See "About the Legend" overleaf.) 


\section{About the legend}

Visual balance is important to illuminated contour maps, and accomplished by having approximately the same area covered by black contours as by white contours. Tanaka's method varies placement of black and white contours and their thickness as a function of aspect only. If all aspect directions are represented equally in an area, the area covered by black and white contours will be equal. This balance is reflected in Figure A-1, which shows a cosine function for $\theta_{\mathrm{T}}$, with line thickness varying for all potential values of $\theta_{\mathrm{T}}$ between $0^{\circ}$ and $360^{\circ}$.

Visual balance is not as simple to achieve using the modified Tanaka method described in this paper. This method varies placement of black and white contours and their thickness as a function of a hill-shading value, traditionally used for tonal variations over areas of shaded relief maps. The hill-shading value varies between $0^{\circ}$ and $90^{\circ}$, with $0^{\circ}$ corresponding to the highest hill-shading value and $90^{\circ}$ corresponding to the lowest hill-shading value (Figure A-2). The central hill-shading value does not occur at $45^{\circ}$ but, rather, at $60^{\circ}$.

The transition from grayness of lines to thickness of black and white lines for illuminated contours requires that an angle be chosen that divides black from white contours. In our study, we used $\theta$ $=45^{\circ}$, an angle which roughly divides our lengths of contours equally between black and white. This value of $\theta=45^{\circ}$ is the result of using an illumination vector with an inclination from horizontal $45^{\circ}$ and a predominance of gently sloping topography. Length of black and white contours has been divided approximately equally; however, this does not ensure that areas covered by black and white contours will be equal.

Black and white contours change thickness in a non-symmetric manner moving away from the central value of $45^{\circ}$. This asymmetry is illustrated in Figure A-3. Contour lines associated with values of $\theta$ between $40^{\circ}$ and $45^{\circ}$ will be the thinnest white contours, and those associated with values of $\theta$ between $45^{\circ}$ and $50^{\circ}$ will be the thinnest black contours. Also, contours must thicken towards $\theta=0$ and $\theta=90$. One method to meet these requirements would be to calculate $\cos (\theta)$, subtract the value of $\cos (45)$, and normalize all thickness to the thickness value of the thinnest contours. This method, displayed in Figure A-3, shows that white lines near $\theta=90$ would be more than twice as thick as black lines near $\theta=0$. Thus, although approximately equal in total lengths, black contours would

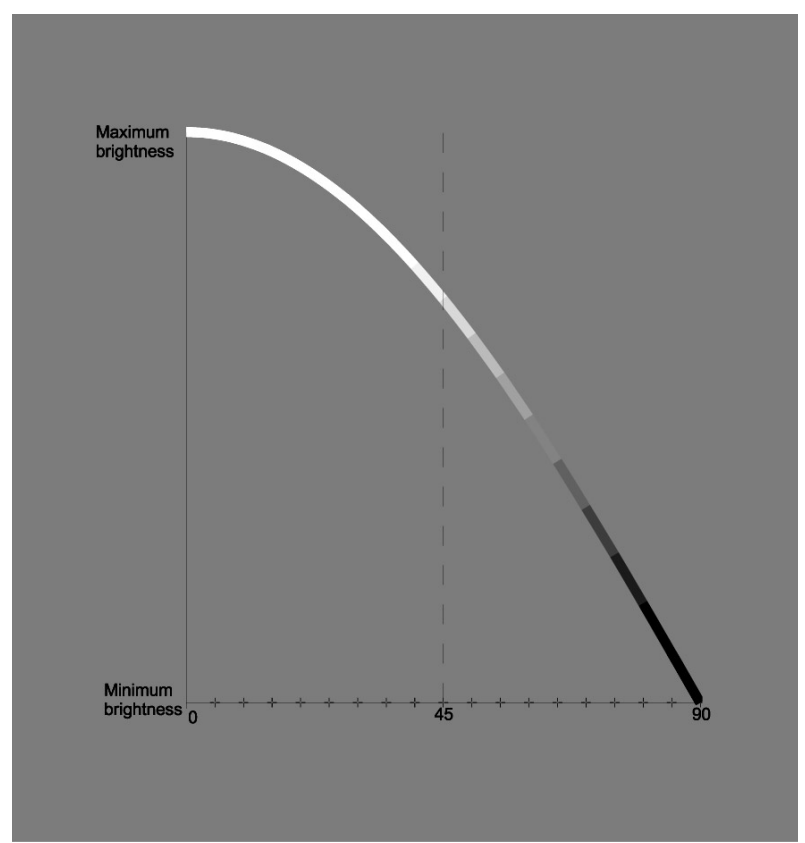

Figure A-2. The analytical hillshading method for assigning brightnesss to line segments based on $\cos (\theta)$. $\theta$ can only have values between $0^{\circ}$ and $90^{\circ}$ and is based on an angle defined by the surface normal and illumination vector.

cover a greater area than white contours with this method.

It is possible to create more symmetrical contours from the angle $\theta$ in a number of ways. The method we used was to calculate the line thickness as a function of $\cos (2 \theta)$ (Figure A-4). With this function, black and white contours change thickness symmetrically away from the thinnest contours in a fashion similar to Tanaka's method. Line thick- 


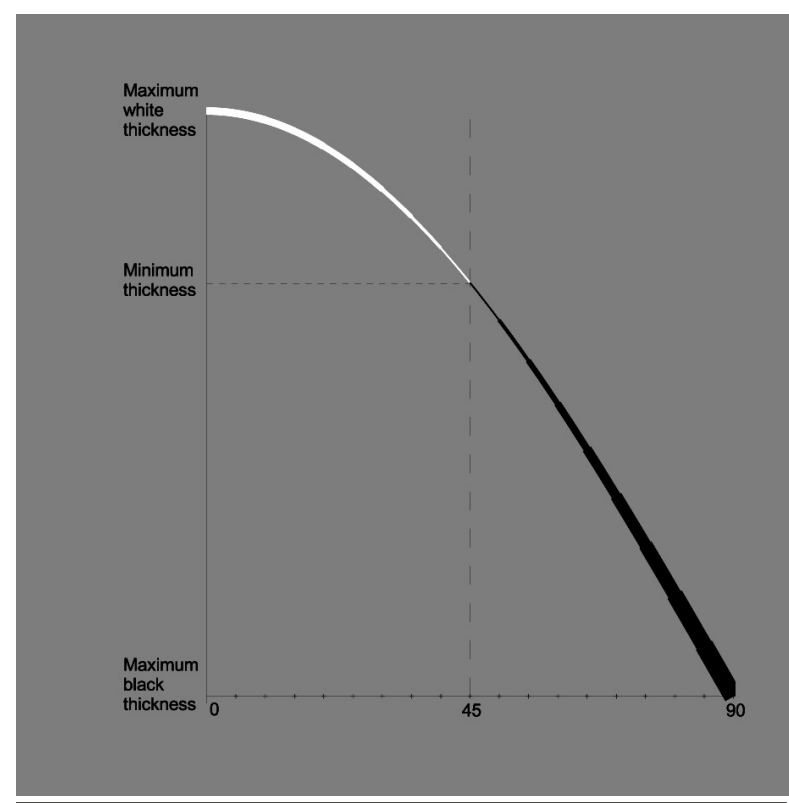

Figure A-3. Black and white line thickness based on cos $(\theta)$. Due to the asymmetry of the cosine function between $0^{\circ}$ and $90^{\circ}$, the resulting black line is thicker than the white line. This would result in a illuminated contour map out of visual balance.

\begin{tabular}{|c|c|c|}
\hline $\boldsymbol{\theta}$ & $\cos (\mathbf{2 q})$ & Thickness $(\mathbf{p t})$ \\
\hline 90 & -1.000000 & -2.88 \\
\hline 85 & -0.984808 & -2.84 \\
\hline 80 & -0.939693 & -2.71 \\
\hline 75 & -0.866025 & -2.49 \\
\hline 70 & -0.766044 & -2.21 \\
\hline 65 & -0.642788 & -1.85 \\
\hline 60 & -0.500000 & -1.44 \\
\hline 55 & -0.342020 & -0.98 \\
\hline 50 & -0.173648 & -0.50 \\
\hline 45 & 0.000000 & NA \\
\hline 40 & 0.173648 & 0.50 \\
\hline 35 & 0.342020 & 0.98 \\
\hline 30 & 0.500000 & 1.44 \\
\hline 25 & 0.642788 & 1.85 \\
\hline 20 & 0.766044 & 2.21 \\
\hline 15 & 0.866025 & 2.49 \\
\hline 10 & 0.939693 & 2.71 \\
\hline 5 & 0.984808 & 2.84 \\
\hline 0 & 1.000000 & 2.88 \\
\hline
\end{tabular}

Table A-1. Relationship between black and white contour thickness values and the angle $\theta$. Positive numbers represent white contours and negative numbers represent black contours. [Thickness is given in points].

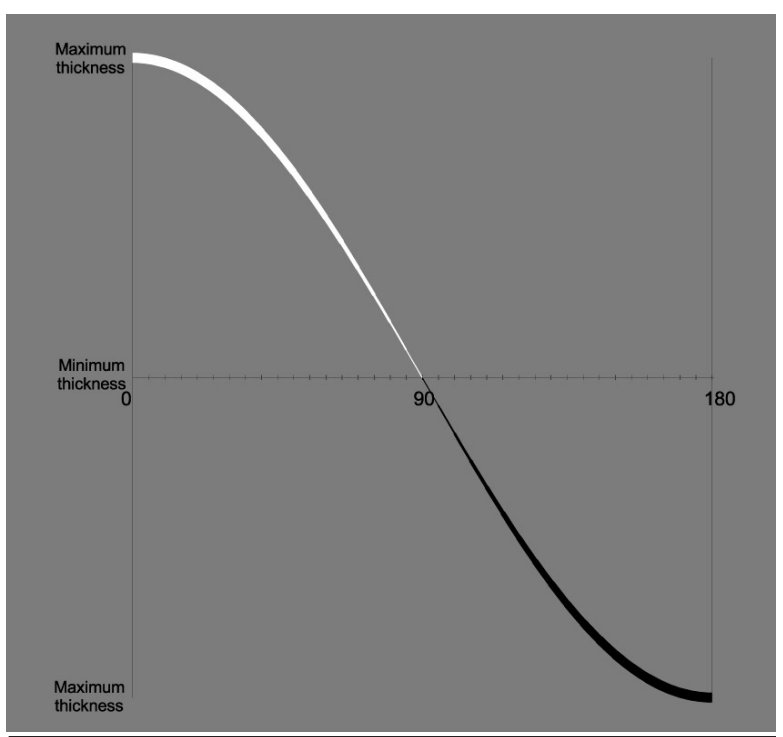

Figure A-4. Black and white line thickness based on cos $(2 \theta)$. The symmetry of this function between $0^{\circ}$ and $180^{\circ}$ results in visual balance, as well matching the thickness patterns of Tanaka's method presented in Figure A-1.

ness values used in this paper are presented in Table A-1. The final column normalizes all values so that the thinnest contour has a thickness of 0.5 pt. Positive numbers represent white contours and negative numbers represent black contours. The shaded portion of the table accounts for 99.7 percent of the line segments by length in this study. 